\title{
Planning, Interface, Decision Making and Sustainability: Common for Engineering, Project, and Production Managers
}

\author{
Kassim Gidado \\ Editor
}

Editorial

Available online November 30, 2013

Programme duration, constraints criticality, managing diverse stakeholders, strategic decision making, and sustainability are common areas of interest in Engineering management, Project management and Production Management. This journal's edition offers a contribution to knowledge and/or novel arguments or ideas, models, methods and procedures to contribute to the fields of research in each of these fields.

Czarnigowska and Sobotka suggest that there are many methods that use cost records of completed projects to predict cost of new schemes, unfortunately there seem to be limited similar methods for predicting duration of new schemes especially at the very early project stage. Their paper identified the factors affecting duration of public road projects and established the potentiality of utilising historic data to forecast durations of similar projects by using simple regression models.

Nguyen and Chua revealed that criticality in a network should not be limited to the critical path alone; there could be a criticality of constraint in the sequence that may lie between non-critical activities. These sequence-criticality occurrences should not be overlooked for effective project schedule controlling. They argued that constraints management must involve activity-critical constraints and sequence-critical constraints but it could disregard non-critical constraints which they consider as redundant constraints. The paper developed a constraint criticality identification procedure that provides a deeper understanding of criticality of schedule constraints to enable project planners choose appropriate strategies to achieve better project performance.

With the increase in using subcontracting in manufacturing and construction industries, White and Marasini drew the attention of engineering, production, and project managers to the need for managing the interface between main and sub-contractors for successful outcomes. Their work focused on the construction industry and highlighted how lack of trust, adverse relationships and culture are influencing delivery of projects. Over the years, the Latham (1994), the Egan (1998) and the Wolstenholme (2009) reports highlighted that the construction industry requires radical change but progress to embrace such change is slow. White and Marasini suggest early involvement of subcontractors, sufficient bidding time, proactive coordination with efficient communication and good site management provided by the main contractor are the crucial factors that can contribute to bringing about the change that the industry needs.

Elnaas et al showed that for the housing industry to meet the numerous demands required of it, the use of offsite manufacturing (OSM) as a strategy is now a necessity. They identified that inappropriate decision making is the reason why there is low uptake despite the advancement of the technology and the tried and tested successful experiences of other industries such as automobile and aircraft. The key contribution of this paper is the identification of the drivers, constraints and factors surrounding the decision to use OSM as a construction strategy for house building.

Lifecycle sustainability is now a core management requirement for any scheme development, delivery and use. Gadakari et al. suggests the use of intelligent buildings to achieving total sustainability. Their work reviews the theoretical concepts of intelligence and sustainability in the built environment. It demonstrated how intelligent building systems improve sustainability rating in environmental, economic and social fronts. They identified the five main barriers to the use of intelligent building systems in modern buildings.

The five papers included in this issue offered an opportunity for the research community to better understand the problems and issues in these diverse fields and suggested new ideas on how the industry can improve performance.

\section{References}

Latham, M. (1994). Constructing the Team - "The Latham Report." London: Department of the Environment. ISBN 9780117529946.

Egan, J. (1998). Rethinking Construction: Report of the Construction Task Force. London: HMSO.

Wolstenholme, A. (2009). Never Waste a Good Crisis: a Review of Progress Since Rethinking Construction and Thoughts for Our Future. London: Constructing Excellence. 